ARTICLE

\title{
Pressure induced metallization with absence of structural transition in layered molybdenum diselenide
}

\author{
Zhao Zhao ${ }^{1, \star}$, Haijun Zhang ${ }^{1,2, \star}$, Hongtao Yuan ${ }^{3,4, \star}$, Shibing Wang ${ }^{4,5}$, Yu Lin ${ }^{5}$, Qiaoshi Zeng 6,7 , Gang Xu ${ }^{3}$, \\ Zhenxian Liu ${ }^{8}$, G.K. Solanki ${ }^{9}$, K.D. Patel ${ }^{9}$, Yi Cui ${ }^{3,4}$, Harold Y. Hwang ${ }^{3,4}$ \& Wendy L. Mao ${ }^{4,5}$
}

Layered transition-metal dichalcogenides have emerged as exciting material systems with atomically thin geometries and unique electronic properties. Pressure is a powerful tool for continuously tuning their crystal and electronic structures away from the pristine states. Here, we systematically investigated the pressurized behavior of $\mathrm{MoSe}_{2}$ up to $\sim 60 \mathrm{GPa}$ using multiple experimental techniques and ab-initio calculations. MoSe $e_{2}$ evolves from an anisotropic two-dimensional layered network to a three-dimensional structure without a structural transition, which is a complete contrast to $\mathrm{MoS}_{2}$. The role of the chalcogenide anions in stabilizing different layered patterns is underscored by our layer sliding calculations. MoSe 2 possesses highly tunable transport properties under pressure, determined by the gradual narrowing of its band-gap followed by metallization. The continuous tuning of its electronic structure and band-gap in the range of visible light to infrared suggest possible energyvariable optoelectronics applications in pressurized transition-metal dichalcogenides.

\footnotetext{
${ }^{1}$ Department of Physics, Stanford University, Stanford, CA 94305, USA. ${ }^{2}$ National Laboratory of Solid State Microstructures and Department of Physics, Nanjing University, Nanjing 210093 China. ${ }^{3}$ Geballe Laboratory for Advanced Materials, Stanford University, Stanford, CA 94305, USA. 4 Stanford Institute for Materials and Energy Sciences, SLAC National Accelerator Laboratory, Menlo Park, CA 94025, USA. ${ }^{5}$ Department of Geological Sciences, Stanford University, Stanford, CA 94305, USA. ${ }^{6}$ HPSynC, Geophysical Laboratory, Carnegie Institution of Washington, Argonne, IL 60439, USA. ${ }^{7}$ Center for High Pressure Science and Technology Advanced Research, Shanghai 201203, China. ${ }^{8}$ Geophysical Laboratory, Carnegie Institution of Washington, Washington, DC 20015, USA. ${ }^{9}$ Department of Physics, Sardar Patel University, Vallabh Vidyanagar, Gujarat 388120, India. ${ }^{*}$ These authors contributed equally to this work. Correspondence and requests for materials should be addressed to Z.Z. (email: zhaozhao@stanford.edu).
} 
T ransition-metal dichalcogenides (TMDs) 2H-MX $(\mathrm{M}=\mathrm{Mo}, \mathrm{W}$, and etc, $\mathrm{X}=\mathrm{S}, \mathrm{Se}$, and $\mathrm{Te})$ have recently attracted intense scientific and engineering interest because of their ease of fabrication and unique electronic structure ${ }^{1-16}$. TMDs have strong chemical bonding within each $\mathrm{X}-\mathrm{M}-\mathrm{X}$ trilayer and weak van der Waals (vdW) interaction between neighbor trilayers in their crystal structures. This quasi two-dimensional (2D) nature grants TMDs facile three-dimensional (3D) to $2 \mathrm{D}$ crossover through methods like exfoliation $1,2,4,5,8,9,12$. Band structure engineering on TMDs allows ones to explore exotic condensed matter phenomena and develop many potential applications. For example, the modification of their band structures from indirect-band-gap to direct-band-gap provides insights into opto-electronics and valley electronics ${ }^{11,14-18}$. So far, the electronic structure engineering of TMDs has mainly been achieved through the following experimental methods: (i) applying electrical field to control the spin splitting and freedom of electrons ${ }^{14,16}$, (ii) utilizing quantum confinement with samples thinning down into monolayers of $\mathrm{MX}_{2}^{11,15,17,18}$, and (iii) employing stress or strain (by bending or stretching the thin films or employing substrates with different lattice constants) ${ }^{19,20}$, as suggested by calculations ${ }^{18,21-26}$.

Compared with the three methods mentioned above, high pressure is a powerful way to induce dramatic changes in their crystal structures and electronic structures ${ }^{27,28}$. This qualifies high pressure as a desirable approach to explore the tunability of TMDs. In particular, the ability to continuously tune the crystal and electronic structures away from the pristine states is crucial to a wide array of applications, e.g., electromechanical devices, energy-variable opto-electronics, and energy-variable photovoltaics. Various pressure-induced electronic evolutions such as semiconductor to metal transitions have been observed in different materials ${ }^{29-31}$. However, many of these electronic transitions were accompanied by first-order structural transitions. A pressure-induced first-order structural transition, by definition, involves a discontinuous change in the volume (of the unit cell). And the corresponding discontinuity in its electronic structure could limit the energy tunability for potential opto-electronic or photovoltaic applications. To overcome this challenge, we need to discover TMDs with continuous structural and electronic response.

Previous high pressure studies on $\mathrm{MoS}_{2}$ clearly demonstrated that a first-order structural transition $\left(2 \mathrm{H}_{\mathrm{c}}-\mathrm{MoS}_{2}\right.$ to $\left.2 \mathrm{H}_{\mathrm{a}}-\mathrm{MoS}_{2}\right)$ occurred close to metallization ${ }^{32-36}$. This transition may relate to the vdW interactions in between neighbor S-Mo-S trilayers ${ }^{36}$. To prevent the $2 \mathrm{H}_{\mathrm{c}}$ to $2 \mathrm{H}_{\mathrm{a}}$ transition, the substitution of chalcogenide anions in $\mathrm{MoS}_{2}$ is a potential route. Because $\mathrm{Se}^{2-}$ has broader electron orbitals than $S^{2-}$ s that lead to stronger interlayer interactions, $\mathrm{MoSe}_{2}$ may have totally different high pressure behavior. Experimentally, the structural and electronic behavior of compressed $\mathrm{MoSe}_{2}$ remains to be fully explored ${ }^{37-39}$. Interestingly, recent calculations have predicted that $\mathrm{MoSe}_{2}$ metallizes between 28 to $40 \mathrm{GPa}$ while preserving the $2 \mathrm{H}_{c}$ structure $^{40}$.

In our work, high pressure up to $\sim 60 \mathrm{GPa}$ was generated by a diamond anvil cell (DAC). X-ray diffraction (XRD) data and Raman spectra data indicate that $\mathrm{MoSe}_{2}$ preserves the $2 \mathrm{H}_{c}$ structure without any structural transition. Ab-initio calculations for modeling the layer sliding process are presented to understand the contrasting behavior between $\mathrm{MoSe}_{2}$ and $\mathrm{MoS}_{2}$, and further predict the structural stability of $\mathrm{MoTe}_{2}$. Infrared (IR) spectra data and temperature-dependent electrical resistivity data demonstrate the highly tunable transport properties of $\mathrm{MoSe}_{2}$. Electronically, both experiments and calculations show that pressure strongly modulates its band structure from semiconducting to metallic.

\section{Results}

XRD and Raman spectroscopy. The experimental set-up of the DAC is shown in Fig. 1a. Under compression, all XRD peaks for $\mathrm{MoSe}_{2}$ continuously shift to larger $2 \theta$ (smaller $d$-spacing) and no new peak is observed (Supplementary Fig. 1 and Supplementary Note 1). Decompression of the sample shows the shifts of all peaks are reversible. All patterns are consistent with the $2 \mathrm{H}_{\mathrm{c}}$ structure and representative Rietveld refinements are shown in Supplementary Fig. 2 and Supplementary Table 1. The absence of a first-order structural transition is further supported by equation of state (EOS) data in Fig. 1b and normalized cell parameters versus pressure data in Fig. 1c, as neither of them exhibits any discontinuity. To fit the pressure-volume relation, a third-order Birch-Murnaghan (BM) EOS is employed. With unitcell volume $V_{0}$ fixed at the experimental value of $120.8 \AA^{3}$, the fitting yields a bulk modulus $B_{0}=62(1) \mathrm{GPa}$ and a derivative of bulk modulus $B^{\prime}=5.6(1)$. The relatively large value of $B^{\prime}$ suggests a strong change of volume compressibility under pressure.

At ambient conditions, the structure of $2 \mathrm{H}_{\mathrm{c}}-\mathrm{MoSe}_{2}$ features the $\mathrm{X}-\mathrm{M}-\mathrm{X}$ triple layers linked via vdW forces (Fig. 2a,b) ${ }^{41,42}$. During the initial compression, cell parameter $c$ is much more compressible than $a$ due to the weak vdW interactions in between Se-Se layers along $c$ (Fig. 1c), while higher pressure allows them to have nearly isotropic contractions. At the highest pressure $\sim 60 \mathrm{GPa}, a$ and $c$ reduce by $\sim 10$ and $\sim 15 \%$ respectively. The gradual closure of the vdW gap is suggested by tracking the ratio of Se-Mo layer distance to Se-Se layer distance, where it drops fast at low pressure but decreases much slower at high pressure (Supplementary Fig. 3). In addition, we measured the Raman spectra under pressure (Supplementary Fig. 4 and Supplementary Note 2). See Fig. 1d, the vibrational modes $A_{1 g}$ and $E_{2 g}$, and the spacing between them shift successively under pressure. These observations indicate that the crystal structure of $\mathrm{MoSe}_{2}$ continuously evolves from a quasi $2 \mathrm{D}$ structure to an isotropic $3 \mathrm{D}$ one without a structural transition.

Structural calculations. Our XRD and Raman results appear to be surprising-At ambient conditions, $\mathrm{MoS}_{2}$ and $\mathrm{MoSe}_{2}$ are iso-structural in crystal structures and possess highly similar electronic structures, and it is therefore natural to assume that the $2 \mathrm{H}_{\mathrm{c}}$ to $2 \mathrm{H}_{\mathrm{a}}$ transition ${ }^{32-35}$ would also occur in $\mathrm{MoSe}_{2}$. Nonetheless, $2 \mathrm{H}_{\mathrm{a}}$ structure does not fit the XRD patterns of $\mathrm{MoSe}_{2}$ in the entire pressure region studied in this work. Though bearing highly similar Mo-Se chemical environments and the same space group $\left(\mathrm{P6}_{3} / \mathrm{mmc}\right), 2 \mathrm{H}_{\mathrm{a}}$ structure and $2 \mathrm{H}_{\mathrm{c}}$ structure have distinct structural topologies. In $2 \mathrm{H}_{\mathrm{a}}$ structure Mo occupies a $2 \mathrm{~b}$ Wyckoff position while in $2 \mathrm{H}_{\mathrm{c}}$ structure it occupies a $2 \mathrm{c}$ Wyckoff position. Also, the two adjacent units of X-Mo-X triple layers have contrasting stacking patterns in $2 \mathrm{H}_{\mathrm{c}}$ and $2 \mathrm{H}_{\mathrm{a}}$ (shown in Fig. 2a-d). To seek theoretical support of the structural stability of $2 \mathrm{H}_{\mathrm{c}}-\mathrm{MoSe}_{2}$, we performed two sets of ab-initio calculations. We first confirmed that $2 \mathrm{H}_{\mathrm{c}}-\mathrm{MoSe}_{2}$ is more stable than $2 \mathrm{H}_{\mathrm{a}}-$ $\mathrm{MoSe}_{2}$, based on the experimental unit cell at the highest pressure $\sim 60 \mathrm{GPa}$. This is consistent with recent calculations showing the enthalpy difference between $2 \mathrm{H}_{\mathrm{a}}$ and $2 \mathrm{H}_{\mathrm{c}}$ of $\mathrm{MoSe}_{2}$ keeps increasing from ambient pressure up to at least $130 \mathrm{GPa}^{40}$. We then calculated the cell parameters at different volumes based on $2 \mathrm{H}_{\mathrm{c}}-\mathrm{MoSe}_{2}$ and the results agree well with our experimental data (Supplementary Fig. 5 and Supplementary Note 3). Note that the small discrepancy at low pressure comes from the limitations of $a b$-initio calculations in describing the vdW interaction.

In order to understand the contrasting structural behavior between $\mathrm{MoS}_{2}{ }^{32-35}$ and $\mathrm{MoSe}_{2}{ }^{40}$, and see whether there is a predictable trend in TMDs, we further carried out layer sliding simulations for $\mathrm{MoS}_{2}, \mathrm{MoSe}_{2}$, and $\mathrm{MoTe}_{2}$ at $\sim 20 \mathrm{GPa}$. The side 

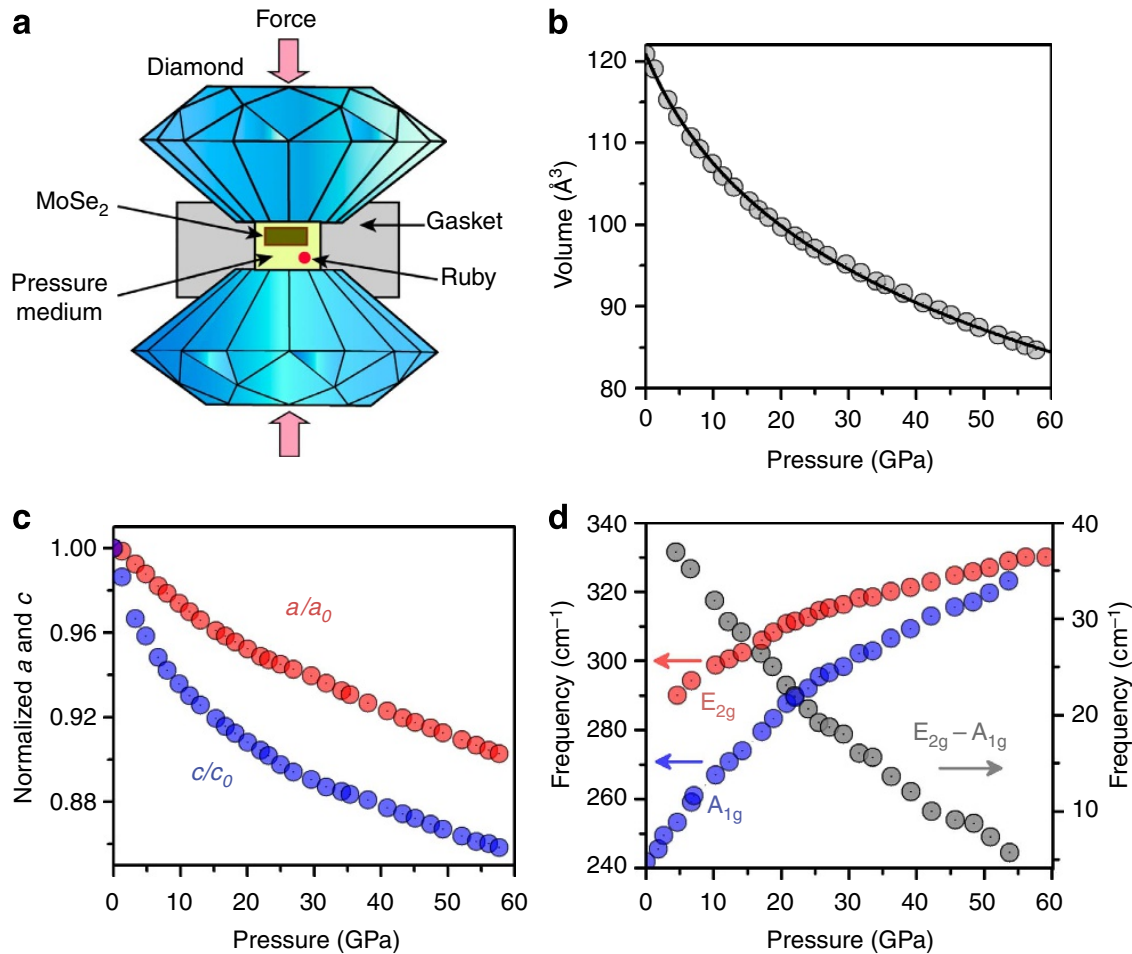

Figure 1 | Experimental set up and structural and vibrational responses under pressure. (a) Schematic of the high pressure DAC set up. (b) Pressurevolume data from XRD measurement and the curve represents a third-order BM-EOS fitting. (c) Normalized cell parameters $a / a_{0}$ and $c / c_{0}$ versus pressure. The error bars given by EXPGUI-GSAS are smaller than the size of the markers. (d) Evolution of vibrational modes $A_{1 g}$ and $E_{2 g}$ and their difference $\left(E_{2 g}-A_{1 g}\right)$ under pressure, measured by Raman spectroscopy.

a

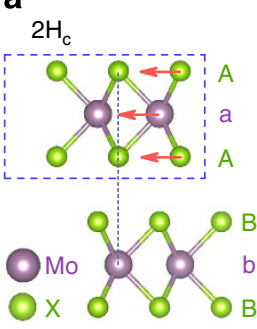

b

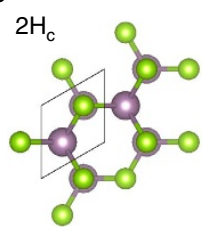

c

$2 \mathrm{H}_{\mathrm{a}}$

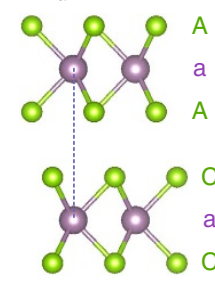

d

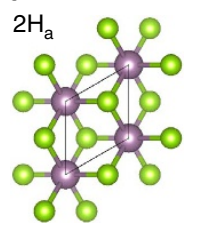

e

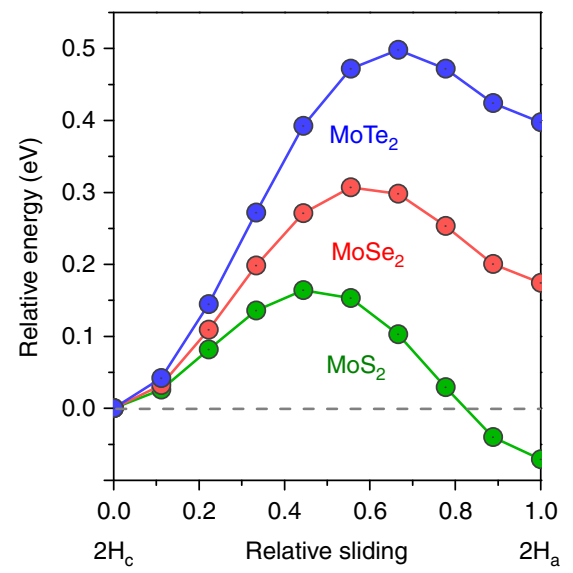

Figure $\mathbf{2}$ | Structural details of $\mathbf{2 H}_{\mathbf{c}}-$ and $\mathbf{2} \mathbf{H}_{\mathbf{a}}-\mathbf{M o X}_{\mathbf{2}}$ and layer sliding calculations. (a) The side view (projected on ac plane) of $2 \mathrm{H}_{\mathrm{c}}$ structure in $\mathrm{MoS}_{2}$ $\mathrm{MoSe}_{2}$ and $\mathrm{MoTe}_{2}$. X represents $\mathrm{S}$, Se and Te. The red arrows represent one sliding path for the $2 \mathrm{H}_{\mathrm{c}}$ to $2 \mathrm{H}_{\mathrm{a}}$ transition, where one unit of $\mathrm{X}-\mathrm{Mo}-\mathrm{X}$ triple layers (marked by a blue box) shifts systematically in $a b$ plane. (b) The top view (projected on $a b$ plane) of $2 \mathrm{H}_{c}$ structure. (d) The side view of $2 \mathrm{H}_{\mathrm{a}}$ structure. (c) The top view of $2 \mathrm{H}_{\mathrm{a}}$ structure. (e) The total energy of $\mathrm{MoS}_{2}, \mathrm{MoSe}_{2}$ and $\mathrm{MoTe}_{2}$ as a function of relative sliding from $2 \mathrm{H}_{\mathrm{c}}$ to $2 \mathrm{H}_{\mathrm{a}}$. The total energies of $2 \mathrm{H}_{\mathrm{c}}$ structures are set to be zero as references, marked by a broken line. For $\mathrm{MoS}_{2}$, the unit cell volume was fixed at experimental data under $20 \mathrm{GPa}^{35}$. After electronic relaxation of the atomic positions, the S-Mo-S layer distance of structure was set to be a constant during the layer sliding. The same procedures were performed on $\mathrm{MoSe}_{2}$ under $23 \mathrm{GPa}$ (our experimental data) and $\mathrm{MoTe}_{2}$ under $20 \mathrm{GPa}$ (theoretical data ${ }^{40}$ ).

and top views of $2 \mathrm{H}_{\mathrm{c}}$-type and $2 \mathrm{H}_{\mathrm{a}}$-type structures are shown in Fig. $2 \mathrm{a}-\mathrm{d}$. The transition from $2 \mathrm{H}_{\mathrm{c}}$ to $2 \mathrm{H}_{\mathrm{a}}$ can be realized by systematically shifting half of the atoms (one unit of X-Mo-X triple layers) in a unit cell. As illustrated in Fig. 2a, we defined one sliding path by the red arrows for this transition. For $\mathrm{MoS}_{2}$, the unit-cell volume is fixed at the experimental value at $20 \mathrm{GPa}^{35}$. After initial relaxation of the atomic positions within the $2 \mathrm{H}_{\mathrm{c}^{-}}{ }^{-}$ type structure, the S-Mo-S layer distances are fixed during the layer sliding. The same procedures are performed for $\mathrm{MoSe}_{2}$ at $23 \mathrm{GPa}$ (using our experimental data) and $\mathrm{MoTe}_{2}$ at $20 \mathrm{GPa}$ (based on previous theoretical data ${ }^{40}$ ). The total energies of $2 \mathrm{H}_{c}$ are set to be zero as the references. Fig. 2e shows the relative energies as a function of the relative layer sliding, i.e., 0 represents $2 \mathrm{H}_{c}$ and 1 represents $2 \mathrm{H}_{\mathrm{a}}$. MoX $\mathrm{X}_{2}$ needs to overcome an energy 
barrier in order to undergo the $2 \mathrm{H}_{\mathrm{c}}$ to $2 \mathrm{H}_{\mathrm{a}}$ transition. The maximum energy barrier is $\sim 0.3 \mathrm{eV}$ for $\mathrm{MoSe}_{2}$ and $\sim 0.5 \mathrm{eV}$ for $\mathrm{MoTe}_{2}$, which are significantly higher than $\sim 0.15 \mathrm{eV}$ for $\mathrm{MoS}_{2}$. More importantly, $2 \mathrm{H}_{\mathrm{a}}-\mathrm{MoSe}_{2}$ and $2 \mathrm{H}_{\mathrm{a}}-\mathrm{MoTe}_{2}$ bear higher energies than the initial $2 \mathrm{H}_{\mathrm{c}}$ structures, which would make this transition unfavorable. However, in the case of $\mathrm{MoS}_{2}$, the $2 \mathrm{H}_{\mathrm{a}}$ structure becomes energetically favorable. Note that the X-Mo-X layer distance is fixed in this set of calculations. Realistically in compressed $\mathrm{MoS}_{2}$, the S-Mo-S distance and unit cell volume drop during the $2 \mathrm{H}_{\mathrm{c}}$ to $2 \mathrm{H}_{\mathrm{a}}$ transition $^{32-35}$, which allows the total change in enthalpy to be continuous at zero temperature.

IR spectroscopy. The lattice response of $\mathrm{MoSe}_{2}$ at high pressure will inevitably change its electronic structure, and thus its optical properties which strongly depend on the electronic structure. Our data shows that $\mathrm{MoSe}_{2}$ undergoes a large electronic evolution where band-gap narrowing is followed by metallization of $\mathrm{MoSe}_{2}$. Fig. 3 shows the measured synchrotron IR spectra and its analysis (details are shown in Supplementary Note 4). Below $16.3 \mathrm{GPa}$, the transmittance spectra (Fig. 3a) look similar, where a transmission window extends from 0.06 to $1.0 \mathrm{eV}$. At pressure above $20.2 \mathrm{GPa}$, the $0.3-1.0 \mathrm{eV}$ region develops into a tilted curve and keeps collapsing into lower energy region, indicating the band-gap's narrowing. At above $40.7 \mathrm{GPa}$ to the highest pressure, nearly zero transmission is observed in between 0.15 to $1.0 \mathrm{eV}$, suggesting the metallization of $\mathrm{MoSe}_{2}$. Another way to interpret the IR data is from the viewpoint of the optical density (OD) $A_{\lambda}$ (see Supplementary Fig. 6 for the plot of OD versus energy). OD or $A_{\lambda}$ is defined as $-\log T$ ( $T$ as the transmittance). It can be easily seen from the energy-pressure-OD map in Fig. $3 b$ that a clear changeover of low OD (in semiconducting state) to high OD (in metallic state) occurs between 20 to $35 \mathrm{GPa}$.

For an indirect-band-gap semiconductor, the absorption coefficient is proportional to the square of the energy difference of the photon energy and band-gap ${ }^{43}$. Using this empirical model for semiconductors, we obtained the indirect-band-gap $E_{\mathrm{g}}$ via linear extrapolations of $\left(h v A_{\lambda}\right)^{1 / 2}$ where $h v$ is the photon energy. A representative extrapolation is shown in Supplementary Fig. 7. The fitted $E_{\mathrm{g}}$ value at $20.2 \mathrm{GPa}$ is $0.4 \mathrm{eV}$. From 20.2 to $35.1 \mathrm{GPa}$, $E_{\mathrm{g}}$ keeps decreasing (see Fig. 3c). From $40.7 \mathrm{GPa}$ to the highest pressure, $E_{\mathrm{g}}$ is nominally zero. We notice that the trend of bandgap decrease at below $41 \mathrm{GPa}$ could not be well described by a linear fitting. The non-linearity is also shown in previous calculations on band-gap's dependence on applied strain ${ }^{23}$. The lack of data points and inaccuracies in optical measurement prevent us from determining the best function for the bandgap - pressure relation. However, as a simple approach to guide eyes, we fit the data with a parabolic curve, which yields $E_{g}=-0.08(2) P+0.0010(3) P^{2}$, indirect-band-gap $\left(E_{\mathrm{g}}\right)$ in unit of $\mathrm{eV}$ and pressure $(P)$ in unit of GPa. The extrapolated band-gap at ambient pressure is $1.6(3) \mathrm{eV}$, which is in good agreement with previous reports ${ }^{18,44,45}$.

Electrical resistivity. We also measured the temperaturedependent resistivity up to $\sim 42 \mathrm{GPa}$ (Supplementary Note 5 and Supplementary Fig. 8). At low pressures (Fig. 4a), the temperature $(T)$ - resistivity $(\rho)$ curves at below $23.4 \mathrm{GPa}$ exhibit negative $d \rho / d T$ throughout all temperatures, indicating the presence of a semiconducting state. From 27.0 to $37.0 \mathrm{GPa}$, the high-temperature region shows positive $d \rho / d T$ whereas the low-temperature region has negative $d \rho / d T$ (see Fig. $4 \mathrm{~b}$ ). At above $41.0 \mathrm{GPa}$, positive $d \rho / d T$ is observed in all temperatures, implying the metallization of $\mathrm{MoSe}_{2}$. A comparison of our room temperature resistivity data on $\mathrm{MoSe}_{2}$ (Fig. 4c) with previous data on $\mathrm{MoS}_{2}$ shows that there are dissimilar trends in between them ${ }^{34,35}$. For $\mathrm{MoS}_{2}$, the room temperature resistivity dropped strongly at below $\sim 15 \mathrm{GPa}$ and then reached a plateau at higher pressure ${ }^{34,35}$, which was related to a first-order structural transition. In sharp contrast, for $\mathrm{MoSe}_{2}$, the decrease of its room temperature resistivity is nearly exponential, fit by $\log (\rho)=1.9(1)-0.134(5) P$, resistivity $(\rho)$ in unit of $\Omega \mathrm{cm}^{-1}$
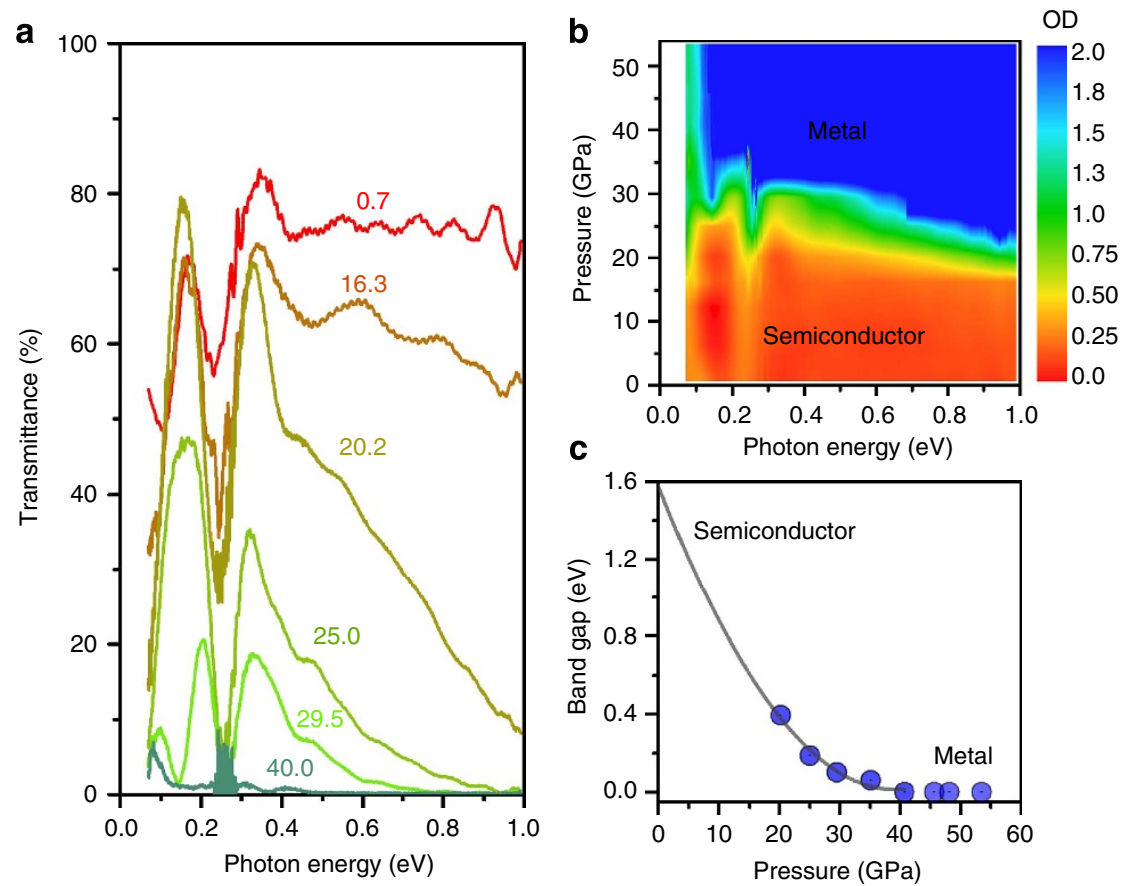

Figure 3 | IR transmittance spectra and analysis. (a) Representative IR transmittance spectra at high pressures, numbers show pressures in unit of GPa. The $0.23-0.28 \mathrm{eV}$ region is obscured by diamond phonon absorptions from the DAC. (b) Pressure - energy - optical density (OD) contour, OD is defined as $-\log (T)$ while $T$ is the transmittance. (c) Evolution of band gap under pressure. Circles are extrapolated indirect band gaps and the curve shows a parabolic fit of the band gap versus pressure. The band gap closure is observed at $\sim 40 \mathrm{GPa}$. 
a

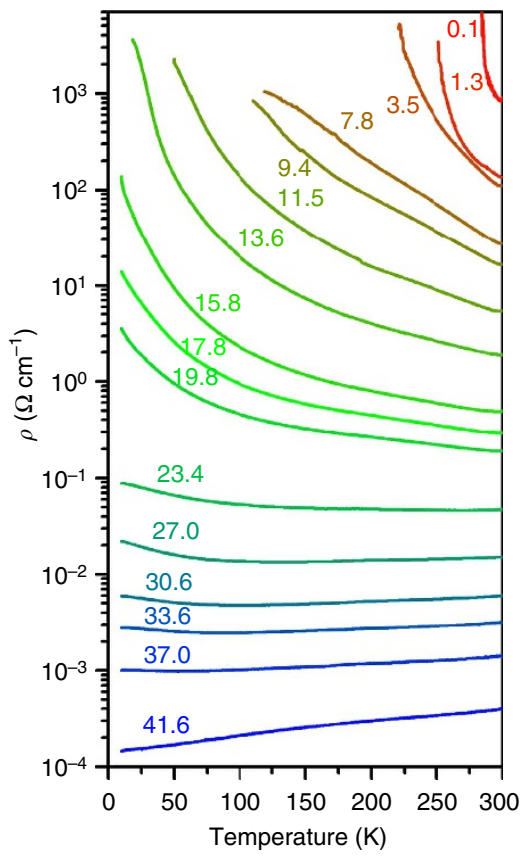

b
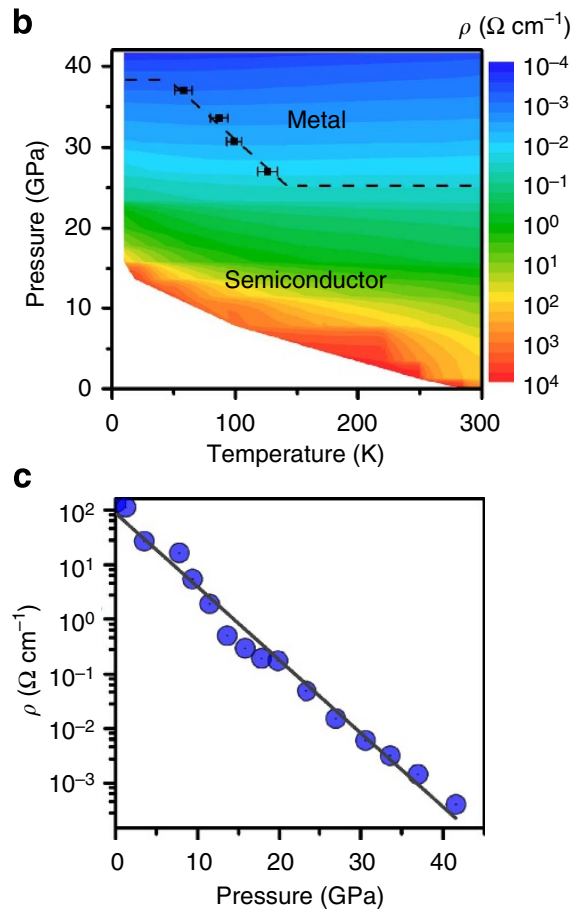

Figure 4 | Temperature-dependent resistivity data and analysis. (a) Temperature-resistivity curves at different pressure, numbers show pressures in GPa. (b) Temperature-pressure-resistivity contour map. (c) Room temperature resistivity versus pressure, the line shows a linear fitting of log $\rho$ versus pressure (equivalent for an exponential fitting of $\rho$ versus pressure).

and pressure $(\mathrm{P})$ in unit of $\mathrm{GPa}$. Pressure allows the room temperature electrical resistivity of $\mathrm{MoSe}_{2}$ to decrease more than six orders of magnitude from ambient to $41.6 \mathrm{GPa}$.

Electronic structure. To better understand the electronic evolution of $\mathrm{MoSe}_{2}$ that determines its highly tunable optical and electrical transport properties, we performed ab-initio calculations on the electronic structure of $\mathrm{MoSe}_{2}$ at four representative pressures. The results undoubtedly demonstrate the pressureinduced band-gap narrowing and metallization. At ambient pressure, seen from Fig. 5a, the band structure is consistent with previous results ${ }^{18,44,45}$. It has a direct-band-gap $E_{\mathrm{K}-\mathrm{K}}(\sim 1.8 \mathrm{eV})$ at $\mathrm{K}$ and an indirect-band-gap $(\sim 1.3 \mathrm{eV})$ that locates in between $\Gamma$ and $\Gamma-\mathrm{K}$ conduction band (CB) valley. The bottom of $\mathrm{CB}$ between $\Gamma$ and $\mathrm{K}$ mainly origins from the Mo $d x y$ and $d x^{2}-y^{2}$ orbitals, and the top of valence bands (VBs) at $\Gamma$ comes from the Mo $d z^{2}$ orbital. Meanwhile, the $d x z$ and $d y z$ dominated CBs are further above from the Fermi-level $\left(E_{\mathrm{F}}\right)$. Higher pressure results in a strong decrease of its indirect-band-gap and induces large movements of the orbitals towards the $E_{F}$. At $23 \mathrm{GPa}$, shown in Fig. 5b, the indirect-band-gap becomes as small as $0.5 \mathrm{eV}$. Albert decreasing, the direct-band-gaps remain at large values, e.g., $E_{\mathrm{K}-\mathrm{K}}$ is $\sim 1.4 \mathrm{eV}$. Remarkably, pressure allows the $d x z$ and $d y z$ orbitals to gain more overlap with Se $p$ orbitals and thus largely widen their band dispersions. In comparison, the $d x y$ and $d x^{2}-y^{2}$ orbitals are less impacted due to smaller overlap with Se $p$ orbitals. As a consequence (see Supplementary Note 6), one $d x z$ and $d y z$ dominated $\mathrm{CB}$ quickly goes down at $\mathrm{K}$ point and forms two $\mathrm{CB}$ valley minimum together with the previous $d x y$ and $d x^{2}-y^{2}$ dominated CB.

Metallic band structures are obtained by further increasing the pressure. For example, seen from Fig. $5 \mathrm{c}$ at $41 \mathrm{GPa}$, there lies density of states across the $E_{\mathrm{F}}$. The $d x y$ and $d x^{2}-y^{2}$ dominated $\mathrm{CB}$ valley minimum crosses below the $E_{\mathrm{F}}$, while the other $\mathrm{CB}$ valley minimum is still slightly above the $E_{\mathrm{F}}$ (see Supplementary Fig. 9). At $58 \mathrm{GPa}$, shown by Fig. 5d, both $\mathrm{CB}$ valley minima cross below the $E_{\mathrm{F}}$. It is worth mentioning that even at as high as $58 \mathrm{GPa}$, the 'indirect' feature of the band structure is still well maintained: although the CBs and VBs overlap in energy range, no direct cross is seen. To be specific, the energy separation at $\mathrm{K}$ is as large as $\sim 0.6 \mathrm{eV}$. Meanwhile, the relative shifts of CBs and VBs generate a number of hole pockets (e.g., at $\Gamma$ and A) and electron pockets (e.g., at K). These pockets may largely affect the lowtemperature electrical and optical transport properties of $\mathrm{MoSe}_{2}$.

\section{Discussions}

Our experiments and calculations clearly demonstrate the absence of structural transition in $\mathrm{MoSe}_{2}$. One empirical understanding of the contrasting behavior in $\mathrm{MoS}_{2}{ }^{32-36}$ and $\mathrm{MoSe}_{2}$ involves the different localizations of $p$ orbitals among chalcogenide anions $\mathrm{S}^{2-}, \mathrm{Se}^{2-}$, and $\mathrm{Te}^{2-}$. The $3 p$ orbitals of $\mathrm{S}^{2-}$ dominate the electronic structure in $\mathrm{MoS}_{2}$ while the $4 p$ and $5 p$ orbitals are primary for $\mathrm{MoSe}_{2}$ and $\mathrm{MoTe}_{2}$ correspondingly. $4 p$ and $5 p$ orbitals are much more delocalized than $3 p$ orbitals, which would give rise to strong interaction within the vdW gaps of $\mathrm{MoSe}_{2}$ and $\mathrm{MoTe}_{2}$ to prevent this sliding process, vice versa for $\mathrm{MoS}_{2}$. We can safely conclude that it is easier for $2 \mathrm{H}_{\mathrm{c}}-\mathrm{MoS}_{2}$ to experience a structural transition through sliding in between neighbor S-Mo-S layers, but this does not apply to $\mathrm{MoSe}_{2}$ or $\mathrm{MoTe}_{2}$. Beside from the effects of chalcogenides anions, the effects of transition metal cation should also be considered in determining the stabilities of layered structures. For example, recent calculations proposed that the interlayer Mo-Mo $d$ electron propagation should be considered in determining the stability of layered structures ${ }^{40}$. More importantly, size effect of different transition metal cations is also expected to change the interlayer interactions. Previous studies on $\mathrm{WS}_{2}$ and $\mathrm{WSe}_{2}{ }^{46-48}$ showed that they experience continuous lattice contractions 

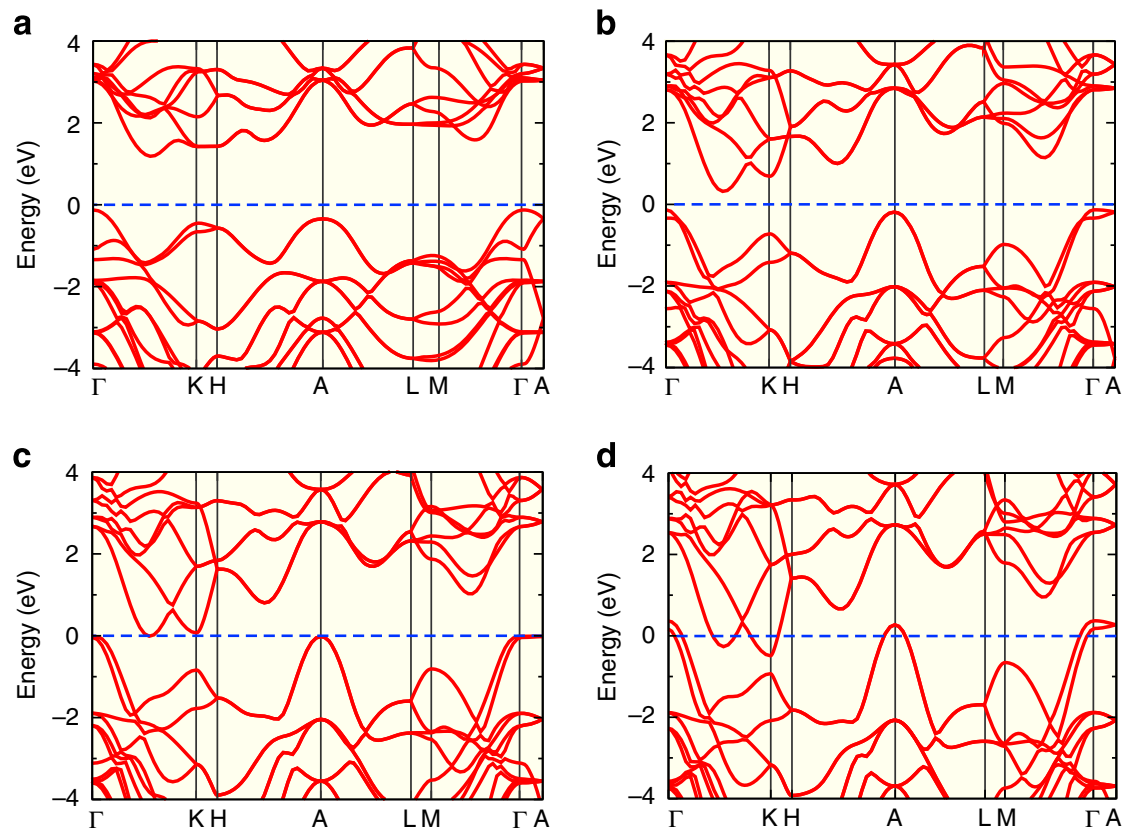

Figure 5 | Calculated band structures of $\mathbf{2 H}_{\mathbf{c}}-\mathbf{M o S e}_{\mathbf{2}}$. (a) Ambient pressure, (b) $23 \mathrm{GPa}$, (c) $41 \mathrm{GPa}$, and (d) $58 \mathrm{GPa}$. Blue dotted line shows the Fermi level $\left(E_{\mathrm{F}}\right)$.

under pressure. $\mathrm{W}^{2+}$ has broader electron orbitals and may introduce stronger interlayer interactions than $\mathrm{Mo}^{2+}$, which results in the absence of layer sliding in $\mathrm{WS}_{2}$. In a more general perspective, stronger interlayer interactions help stabilize the structures of TMDs and are more likely to yield continuous lattice response under extreme environments such as pressure.

Previous studies have reported many electronic transitions such as insulator to metal or semiconductor to metal transitions in the group of binary chalcogenides, see for example, $\mathrm{Bi}_{2} \mathrm{X}_{3}{ }^{31,49,50}, \mathrm{Sb}_{2} \mathrm{X}_{3}{ }^{51-53}$, and $\mathrm{Ag}_{2} \mathrm{X}^{54-56}$. For structures starting with vdW gaps at ambient conditions, the closure of their vdW gaps is generally accompanied or followed by first-order structural transitions where large structural re-constructions or atomic movements take place ${ }^{43-48}$. However, in the case of $\mathrm{MoSe}_{2}$, the metallization process does not involve any sudden change in the crystal structure, which allows its electronic structure to be continuously tuned. Through multiple experimental techniques combined with ab-initio calculations, we demonstrate that the band-gap of $\mathrm{MoSe}_{2}$ (in the range of visible to IR region) exhibits a strong dependence on pressure. This may allow $\mathrm{MoSe}_{2}$, one representative TMD, to be applied in energy-variable opto-electronics and photovoltaics, although the limitation of sample size $(0.05$ to $0.1 \mathrm{~mm})$ must be taken into account in future investigation.

Compared with others methods of band structure engineering approachable by experiments ${ }^{11-18}$, pressure is the only way to metalize $\mathrm{MoSe}_{2}$ and $\mathrm{MoS}_{2}{ }^{34,35}$. This highlights pressure's dramatic role in tuning the electronic properties of TMDs. Our different aspects of the experimental data show good agreement with the recent calculations on $\mathrm{MoSe}_{2}{ }^{40}$. This further suggests $2 \mathrm{H}_{\mathrm{c}}-\mathrm{MoSe}_{2}$ a suitable material with a concise unit cell for testing and improving first-principles calculations that can be probed by experiments. If future experiments, i.e., by applying non-uniaxial compression, are able break the inversion symmetry of the crystal structure of pressurized $\mathrm{MoSe}_{2}$, the spin and valley electronics of $\mathrm{MoSe}_{2}$ would be largely different when we consider the large shifts of the CBs and VBs $5,12,14,16$. More importantly, the novel scenario of excitonic insulator may be experimentally realizable in $\mathrm{MoSe}_{2}{ }^{40}$, while in $\mathrm{MoS}_{2}$ the complexity of the layer sliding structural transition may prevent the formation of this electronic state ${ }^{32-36}$. For the pressurized metallic $\mathrm{MoSe}_{2}$, its distinguished 'indirect' band structures and electronics state filled with electron holes and pockets await further exploitation in condensed matters physics, i.e., charge density wave or superconductivity may be found in TMDs at higher pressure $35,36,40$.

In conclusion, we comprehensively studied the high pressure behavior of $\mathrm{MoSe}_{2}$ up to $\sim 60 \mathrm{GP}$ through a series of structural, vibrational, optical, and electrical measurements combined with ab-initio calculations. $2 \mathrm{H}_{\mathrm{c}}-\mathrm{MoSe}_{2}$ evolves from an anisotropic $2 \mathrm{D}$ layered structure to an isotropic $3 \mathrm{D}$ one without any sudden structural change under pressure. Our layer sliding calculations highlight the role of the chalcogenide anions in stabilizing either $2 \mathrm{H}_{\mathrm{a}}$ or $2 \mathrm{H}_{\mathrm{c}}$ layered patterns. Electronically, $\mathrm{MoSe}_{2}$ undergoes a semiconductor to metal transition, and correspondingly exhibits highly tunable optical and electrical properties. Upon compression, the 'indirect' feature of its electronic structure is robustly conserved with the appearance of two conduction band minima. The large and continuous tuning of its electronic structure may have potential applications in energy-variable (visible to IR) optoelectronics and photovoltaics.

\section{Methods}

Sample growth. High quality stoichiometric $\mathrm{MoSe}_{2}$ single crystals were grown by direct vapor transport technique ${ }^{39,44,57}$. Elemental Mo and Se (99.9\% purity, purchased from Koch Light Ltd.) of the stoichiometric ratio were sealed in a quartz ampoule at pressure better than $10^{-5}$ Torr. The ampoule was placed in a two-zone horizontal furnace where the temperatures were slowly raised from room temperature to 1,060 and $1,080^{\circ} \mathrm{C}$ for growth zone and source zone respectively. This temperature gradient was then maintained for $\sim 188 \mathrm{~h}$ to produce to single crystal platelets of $\mathrm{MoSe}_{2}$. The shiny and gray crystals have a typical thickness of $\sim 4 \mu \mathrm{m}$ and area of $\sim 3 \mathrm{~mm} \times 3 \mathrm{~mm}$. The purity and homogeneity are checked by electron microprobe analysis.

High pressure experiments. Single crystals of $\mathrm{MoSe}_{2}$ were used for the high pressure IR, Raman, and resistivity measurements. Powders of $\mathrm{MoSe}_{2}$ were grounded from single crystals for the high pressure XRD measurements. Ruby spheres were used for determining pressure for all experiments. Neon was used as the pressure transmitting medium for the XRD and Raman experiments. Angle dispersive XRD experiments were performed at beamline 16-BMD of the Advanced Photon Source (APS), Argonne National Laboratory (ANL). The Rietveld fitting was performed using GSAS-EXPGUI package ${ }^{58}$. The Raman spectra were collected 
using a Renishaw inVia micro Raman system with a $514 \mathrm{~nm}$ laser excitation line. High-pressure IR measurements were conducted at beamline U2A of the National Synchrotron Light Source (NSLS), Brookhaven National Laboratory (BNL). A $\mathrm{MoSe}_{2}$ single crystal was sandwiched between the pressure transmitting medium $(\mathrm{KBr})$ and one side of the culet. Infrared measurements were performed on a Bruker Vertex 80v FT-IR spectrometer coupled to a Hyperion-2000 microscope with a MCT mid-band detector. Fringes in raw IR data were removed by filtering high frequency terms after Fourier transformation. For temperature-dependent four-probe resistivity measurement, cubic BN was used as the insulating layer, and four electrodes were cut from Pt foils. The temperature-dependent sheet resistance of the sample was measured with Van der Paul geometry by cooling down to $10 \mathrm{~K}$ in a liquid helium cryostat. Pressures were measured at room temperature. More details are described in the supplementary information.

$\boldsymbol{A} \boldsymbol{b}$-initio calculations. The Vienna ab-initio Simulation Package ${ }^{59,60}$ was employed to optimize crystal structures and calculate electronic structures with the framework of local density approximation density functional theory ${ }^{61}$. The projector augmented wave ${ }^{62}$ pseudo-potential was used and the kinetic energy cutoff was fixed to $450 \mathrm{eV}$ for all the calculations. For structural calculations in comparison with experiments, the unit cell volume is fixed while the cell parameters and atomic positions are fully relaxed. For band structure calculations, the lattice constants were fixed to be experimental values, and then the atomic positions are fully relaxed. HSE06 hybrid function ${ }^{63}$ was employed to improve the band structure calculations.

\section{References}

1. Novoselov, K. S. et al. Two-dimensional atomic crystals. Proc. Natl. Acad. Sci. 102, 10451-10453 (2005)

2. Mak, K. F., Lee, C., Hone, J., Shan, J. \& Heinz, T. F. Atomically Thin $\mathrm{MoS}_{2}$ : A New Direct-Gap Semiconductor. Phys. Rev. Lett. 105, 136805 (2010).

3. Komsa, H.-P. et al. Two-Dimensional Transition Metal Dichalcogenides under Electron Irradiation: Defect Production and Doping. Phys. Rev. Lett. 109, 035503 (2012)

4. Tongay, S. et al. Thermally driven crossover from indirect toward direct bandgap in 2D semiconductors: $\mathrm{MoSe}_{2}$ versus $\mathrm{MoS}_{2}$. Nano Lett. 12, 5576-5578 (2012).

5. Wang, Q. H., Kalantar-Zadeh, K., Kis, A., Coleman, J. N. \& Strano, M. S. Electronics and optoelectronics of two-dimensional transition metal dichalcogenides. Nat. Nanotechnol. 7, 699-712 (2012).

6. Xiao, D., Liu, G.-B., Feng, W., Xu, X. \& Yao, W. Coupled Spin and Valley Physics in Monolayers of $\mathrm{MoS}_{2}$ and Other Group-VI Dichalcogenides. Phys. Rev. Lett. 108, 196802 (2012).

7. Han, S. W. et al. Controlling Ferromagnetic Easy Axis in a Layered $\mathrm{MoS}_{2}$ Single Crystal. Phys. Rev. Lett. 110, 247201 (2013).

8. Jin, W. et al. Direct Measurement of the Thickness-Dependent Electronic Band Structure of $\mathrm{MoS}_{2}$ Using Angle-Resolved Photoemission Spectroscopy. Phys. Rev. Lett. 111, 106801 (2013)

9. Li, X., Zhang, F. \& Niu, Q. Unconventional Quantum Hall Effect and Tunable Spin Hall Effect in Dirac Materials: Application to an Isolated $\mathrm{MoS}_{2}$ Trilayer. Phys. Rev. Lett. 110, 066803 (2013).

10. Mkhonta, S. K., Elder, K. R. \& Huang, Z.-F. Exploring the Complex World of Two-Dimensional Ordering with Three Modes. Phys. Rev. Lett. 111, 035501 (2013).

11. Qiu, D. Y., da Jornada, F. H. \& Louie, S. G. Optical Spectrum of $\mathrm{MoS}_{2}$ : ManyBody Effects and Diversity of Exciton States. Phys. Rev. Lett. 111, 216805 (2013).

12. Sun, L. et al. Spin-Orbit Splitting in Single-Layer $\mathrm{MoS}_{2}$ Revealed by Triply Resonant Raman Scattering. Phys. Rev. Lett. 111, 126801 (2013).

13. Wang, H., Lu, Z., Xu, S. \& Kong, D. Electrochemical tuning of vertically aligned $\mathrm{MoS}_{2}$ nanofilms and its application in improving hydrogen evolution reaction. Proc. Natl. Acad. Sci. 110, 19701-19706 (2013).

14. Yuan, H. et al. Zeeman-type spin splitting controlled by an electric field. Nat. Phys. 9, 563-569 (2013)

15. Zhang, Y., Chang, T., Zhou, B. \& Cui, Y. Direct observation of the transition from indirect to direct bandgap in atomically thin epitaxial $\mathrm{MoSe}_{2}$. Nat. Nanotechnol. 9, 111-115 (2014).

16. Yuan, H. et al. Generation and electric control of spin-valley-coupled circular photogalvanic current in WSe 2 . Nat. Nanotechnol. 9, 851-857 (2014).

17. Ramasubramaniam, A., Naveh, D. \& Towe, E. Tunable band gaps in bilayer transition-metal dichalcogenides. Phys. Rev. B 84, 205325 (2011).

18. Yun, W. S., Han, S. W., Hong, S. C., Kim, I. G. \& Lee, J. D. Thickness and strain effects on electronic structures of transition metal dichalcogenides: $2 \mathrm{H}-\mathrm{MX}_{2}$ semiconductors $(\mathrm{M}=\mathrm{Mo}, \mathrm{W} ; \mathrm{X}=\mathrm{S}, \mathrm{Se}, \mathrm{Te})$. Phys. Rev. B 85, 033305 (2012).

19. Zhu, C. R. et al. Strain tuning of optical emission energy and polarization in monolayer and bilayer $\mathrm{MoS}_{2}$. Phys. Rev. B 88, 121301(R) (2013).

20. Hui, Y. Y. et al. Exceptional tunability of band energy in a compressively strained trilayer $\mathrm{MoS}_{2}$ sheet. ACS Nano 7, 7126-7131 (2013).
21. Kaplan-Ashiri, I. et al. On the mechanical behavior of $\mathrm{WS}_{2}$ nanotubes under axial tension and compression. Proc. Natl. Acad. Sci. 103, 523-528 (2006).

22. Johari, P. \& Shenoy, V. B. Tuning the Electronic Properties of Semiconducting Transition Metal Dichalcogenides by Applying Mechanical Strains. ACS Nano 6, 5449-5456 (2012).

23. Bhattacharyya, S. \& Singh, A. K. Semiconductor-metal transition in semiconducting bilayer sheets of transition-metal dichalcogenides. Phys. Rev. B 86, 075454 (2012).

24. Peelaers, H. \& Van de Walle, C. G. Effects of strain on band structure and effective masses in $\mathrm{MoS}_{2}$. Phys. Rev. B 86, 241401(R) (2012).

25. Cheiwchanchamnangij, T., Lambrecht, W. R. L., Song, Y. \& Dery, H. Strain effects on the spin-orbit-induced band structure splittings in monolayer $\mathrm{MoS}_{2}$ and graphene. Phys. Rev. B 88, 155404 (2013).

26. Chang, C.-H., Fan, X., Lin, S.-H. \& Kuo, J.-L. Orbital analysis of electronic structure and phonon dispersion in $\mathrm{MoS}_{2}, \mathrm{MoSe}_{2}, \mathrm{WS}_{2}$, and $\mathrm{WSe}_{2}$ monolayers under strain. Phys. Rev. B 88, 195420 (2013).

27. Jayaraman, A. Diamond anvil cell and high-pressure physical investigations. Rev. Mod. Phys. 55, 65-108 (1983).

28. Mujica, A., Rubio, A., Muñoz, A. \& Needs, R. J. High-pressure phases of groupIV, III-V, and II-VI compounds. Rev. Mod. Phys. 75, 863-912 (2003).

29. Arcangeletti, E. et al. Evidence of a Pressure-Induced Metallization Process in Monoclinic $\mathrm{VO}_{2}$. Phys. Rev. Lett. 98, 196406 (2007).

30. Vilaplana, R. et al. High-pressure vibrational and optical study of $\mathrm{Bi}_{2} \mathrm{Te}_{3}$. Phys Rev. B 84, 104112 (2011).

31. Segura, A. et al. Trapping of three-dimensional electrons and transition to twodimensional transport in the three-dimensional topological insulator $\mathrm{Bi}_{2} \mathrm{Se}_{3}$ under high pressure. Phys. Rev. B 85, 195139 (2012).

32. Aksoy, R. et al. X-ray diffraction study of molybdenum disulfide to $38.8 \mathrm{GPa}$ J. Phys. Chem. Solids 67, 1914-1917 (2006).

33. Livneh, T. \& Sterer, E. Resonant Raman scattering at exciton states tuned by pressure and temperature in $2 \mathrm{H}-\mathrm{MoS}_{2}$. Phys. Rev. B 81, 195209 (2010).

34. Nayak, A. P. et al. Pressure-induced semiconducting to metallic transition in multilayered molybdenum disulphide. Nat. Commun. 5, 3731 (2014).

35. Chi, Z.-H. et al. Pressure-Induced Metallization of Molybdenum Disulfide. Phys. Rev. Lett. 113, 036802 (2014).

36. Hromadová, L., Martońák, R. \& Tosatti, E. Structure change, layer sliding, and metallization in high-pressure $\mathrm{MoS}_{2}$. Phys. Rev. B 87, 144105 (2013).

37. Aksoy, R., Selvi, E. \& Ma, Y. X-ray diffraction study of molybdenum diselenide to $35.9 \mathrm{GPa}$. J. Phys. Chem. Solids 69, 2138-2140 (2008).

38. Sugai, S. \& Ueda, T. High-pressure Raman spectroscopy in the layered materials $2 \mathrm{H}-\mathrm{MoS}_{2}, 2 \mathrm{H}-\mathrm{MoSe}_{2}$, and $2 \mathrm{H}-\mathrm{MoTe}_{2}$. Phys. Rev. B 26, 6554-6558 (1982).

39. Dave, M., Vaidya, R., Patel, S. G. \& Jani, A. R. High pressure effect on $\mathrm{MoS}_{2}$ and $\mathrm{MoSe}_{2}$ single crystals grown by CVT method. Bull. Mater. Sci. 27, 213-216 (2004).

40. Rifliková, M., Martoňák, R. \& Tosatti, E. Pressure-induced gap closing and metallization of $\mathrm{MoSe}_{2}$ and $\mathrm{MoTe}_{2}$. Phys. Rev. B 90, 035108 (2014).

41. James, P. B. \& Lavik, M. T. The crystal structure of $\mathrm{MoSe}_{2}$. Acta Crystallogr. 16, 1183-1183 (1963).

42. Wilson, J. A. \& Yoffe, A. D. The transition metal dichalcogenides discussion and interpretation of the observed optical, electrical and structural properties. Adv. Phys. 18, 193-335 (1969).

43. Fox, M. in Opt. Prop. Solids (Oxford Univ. Press, 2010).

44. Mahatha, S. K., Patel, K. D. \& Menon, K. S. R. Electronic structure investigation of $\mathrm{MoS}_{2}$ and $\mathrm{MoSe}_{2}$ using angle-resolved photoemission spectroscopy and ab initio band structure studies. J. Phys. Condens. Matter 24, 475504 (2012).

45. Coehoorn, R. et al. Electronic structure of $\mathrm{MoSe}_{2}, \mathrm{MoS}_{2}$, and WSe 2 . I. Bandstructure calculations and photoelectron spectroscopy. Phys. Rev. B 35, 6195 (1987).

46. Selvi, E., Aksoy, R., Knudson, R. \& Ma, Y. High-pressure X-ray diffraction study of tungsten diselenide. J. Phys. Chem. Solids 69, 2311-2314 (2008).

47. Liu, B. et al. Pressure Induced Semiconductor-Semimetal Transition in $\mathrm{WSe}_{2}$. J. Phys. Chem. C 114, 14251-14254 (2010).

48. Bandaru, N. et al. Structural stability of $\mathrm{WS}_{2}$ under high pressure. Int. J. Mod. Phys. B 28, 1450168-1-10 (2014).

49. Polian, A. et al. Two-dimensional pressure-induced electronic topological transition in $\mathrm{Bi}_{2} \mathrm{Te}_{3}$. Phys. Rev. B 83, 113106 (2011).

50. Zhang, J. et al. Electronic topological transition and semiconductor-to-metal conversion of $\mathrm{Bi}_{2} \mathrm{Te}_{3}$ under high pressure. Appl. Phys. Lett. 103, 052102 (2013).

51. Gomis, O. et al. Lattice dynamics of $\mathrm{Sb}_{2} \mathrm{Te}_{3}$ at high pressures. Phys. Rev. B 84, 174305 (2011).

52. Zhao, J. et al. Pressure-induced disordered substitution alloy in $\mathrm{Sb}_{2} \mathrm{Te}_{3}$. Inorg Chem. 50, 11291-11293 (2011).

53. Bera, A. et al. Sharp Raman Anomalies and Broken Adiabaticity at a Pressure Induced Transition from Band to Topological Insulator in $\mathrm{Sb}_{2} \mathrm{Se}_{3}$. Phys. Rev. Lett. 110, 107401 (2013). 
54. Zhang, J. et al. Impurity level evolution and majority carrier-type inversion of $\mathrm{Ag}_{2} \mathrm{~S}$ under extreme compression: Experimental and theoretical approaches. Appl. Phys. Lett. 103, 082116 (2013).

55. Zhao, Z., Wang, S., Zhang, H. \& Mao, W. L. Pressure induced structural transitions and metallization in $\mathrm{Ag}_{2}$ Te. Phys. Rev. B 88, 024120 (2013).

56. Zhao, Z. et al. Tuning the crystal structure and electronic states of $\mathrm{Ag}_{2} \mathrm{Se}$ : Structural transitions and metallization under pressure. Phys. Rev. B 89, 180102(R) (2014).

57. Agarwal, M. K., Patel, P. D. \& Gupta, S. K. Effect of doping $\mathrm{MoSe}_{2}$ single crystals with rhenium. J. Cryst. Growth 129, 559-562 (1993).

58. Toby, B. H. EXPGUI, a graphical user interface for GSAS. J. Appl. Cryst. 34, 210-213 (2001).

59. Kresse, G. \& Joubert, D. From ultrasoft pseudopotentials to the projector augmented-wave method. Phys. Rev. B 59, 1758-1775 (1999).

60. Kresse, G. \& Hafner, J. Ab initio molecular dynamics for liquid metals. Phys Rev. B 47, 558-561 (1993).

61. Hohenberg, P. \& Kohn, W. Inhomogeneous electron gas. Phys. Rev. 155, 864-871 (1964).

62. Blöchl, P. E. Projector augmented-wave method. Phys. Rev. B 50, 17953-17979 (1994).

63. Heyd, J., Scuseria, G. E. \& Ernzerhof, M. Hybrid functionals based on a screened Coulomb potential. J. Chem. Phys. 124, 8207-8215 (2006).

\section{Acknowledgements}

We thank Y. Xu at Stanford University for useful discussions. We thank S. Tkachev, C. Park, S. Sinogeikin, H. Yan, and Y. Meng at APS for their technical assistance. Z.Z., H.T.Y., Y.L., H.Y.H., Y.C., and W.L.M. are supported by the Department of Energy (DOE), Basic Energy Sciences (BES), Materials Sciences and Engineering Division, under Contract DE-AC02-76SF00515. H.Z. is supported by ARO W911NF-09-1-0508. K.D.P. and G.K.S. are funded by a major research project from University Grants Commission, New Delhi, India. HPCAT operations are supported by DOE-NNSA, DE-NA0001974 and DOE-BES, DE-FG02-99ER45775, with partial instrumentation funding by NSF MRI-1126249. APS is supported by DOE-BES, DE-AC02-06CH11357. U2A is supported by COMPRES under NSF Cooperative Agreement EAR 11-57758 and DOE-NNSA, DE-FC03-03N00144, CDAC. NSLS is supported by DOE-BES, DE-AC02-98CH10886.

\section{Author contributions}

Z.Z., H.Z., H.T.Y., and W.L.M. designed the project. G.K.S. and K.D.P. synthesized the samples. Z.Z., H.T.Y., S.W., Y.L., Q.Z., and Z. L. conducted the experiments. H.Z. performed the calculations. Y.C., H.Y.H, and W.L.M. supervised the project and all authors contributed to data discussions. Z.Z., H.Z., and H.T.Y., wrote the paper with inputs from all others.

\section{Additional information}

Supplementary Information accompanies this paper at http://www.nature.com/ naturecommunications

Competing financial interests: The authors declare no competing financial interests.

Reprints and permission information is available online at http://npg.nature.com/ reprintsandpermissions/

How to cite this article: Zhao, Z. et al. Pressure induced metallization with absence of structural transition in layered molybdenum diselenide. Nat. Commun. 6:7312 doi: $10.1038 /$ ncomms8312 (2015).

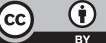

This work is licensed under a Creative Commons Attribution 4.0 International License. The images or other third party material in this article are included in the article's Creative Commons license, unless indicated otherwise in the credit line; if the material is not included under the Creative Commons license, users will need to obtain permission from the license holder to reproduce the material. To view a copy of this license, visit http://creativecommons.org/licenses/by/4.0/ 